\title{
Digital templating in total hip arthroplasty using contralateral healthy hip results in decreased accuracy
}

\author{
Ittai Shichman, Or Shaked, Samuel Morgan, Simon Garceau, Nimrod Snir, Yaniv Warschawski
}

\begin{abstract}
From the Adult Reconstruction Unit, Division of Orthopedics, Tel-Aviv Sourasky Medical Center, Tel-Aviv, affiliated to the Sackler Faculty of Medicine Tel-Aviv University, Tel-Aviv Israel
\end{abstract}

The accuracy of pre-operative digital templating for total hip arthroplasty (THA) using the diseased versus unaffected contralateral joint remains unclear. As such, we devised a study to compare templating precision between the operated hip joint versus the healthy side for patients with osteoarthritis (OA). The study hypothesis was that preoperative templating accuracy of THA on the ipsilateral diseased hip joint would be higher compared to the contralateral healthy hip in patients with OA. We retrospectively reviewed 100 patients who underwent THA for unilateral OA at our center from January 2018 to January 2020. Retrospective preoperative digital templating was performed separately on both the operated hip joint and the healthy contralateral hip joint by a single surgeon who was blinded by the in-situ components sizes. Accuracy of each group was compared to the implanted components. Assessment of the $\mathbf{1 0 0}$ included cases demonstrated superior acetabular component size prediction when templating was performed using the diseased hip compared to the healthy contralateral side $(68.0 \%$ versus $\mathbf{5 1 . 0 \%}, \mathbf{p}<\mathbf{0 . 0 0 1})$. No differences between the cohorts were found regarding templating accuracy of femoral stem sizes $(\mathbf{7 2 . 0} \%$ and $69.0 \%, p=0.375)$ or neck offset $(73.0 \%$ and $69.0 \%, p=0.289)$. Templating acetabular cup size using the ipsilateral diseased hip is more accurate than using the contralateral healthy hip in patients with unilateral OA.

Disclosure of interest: The Authors declare that there is no conflict of interest.

Funding: This research did not receive any specific grant from funding agencies in the public, commercial, or not-forprofit sectors.
Keywords : Total hip arthroplasty ; digital templating ; osteoarthritis ; calibration ; TraumaCAD.

\section{INTRODUCTION}

Preoperative templating in total hip arthroplasty (THA) has proven to be helpful in the selection of proper implant sizes, facilitating optimal component alignment, reducing surgical times and risk of potential complications (1-5).

Over the past decade, digital templating has largely replaced conventional acetate templating

Ittai Shichman ${ }^{1}, \mathrm{MD}$,

Or Shaked ${ }^{1}, \mathrm{MD}-\mathrm{MPH}$,

Samuel Morgan², MD,

Simon Garceau ${ }^{3}, \mathrm{MD}$,

Nimrod SNIR ${ }^{1}, \mathrm{MD}$,

Yaniv Warschawski ${ }^{1}, \mathrm{MD}$

${ }^{1}$ Adult Reconstruction Unit, Division of Orthopedics, TelAviv Sourasky Medical Center, Tel-Aviv, affiliated to the Sackler Faculty of Medicine Tel-Aviv University, Tel-Aviv Israel.

${ }^{2}$ Sackler School of Medicine, Tel-Aviv University, Tel-Aviv, Israel.

${ }^{3}$ NYU Langone Orthopedic Hospital, NYU Langone Health, New-York, USA.

Correspondence : Or Shaked, Division of Orthopedics, TelAviv Sourasky Medical Center, 6 Weizman Street, Tel-Aviv, 6423906 Israel.

Email : orshaked@gmail.com

- 2021, Acta Orthopædica Belgica.

Acta Orthopædica Belgica, Vol. 87 - 3 - 2021 
methods as the standard modality used in preoperative planning (3). A combination of digital radiography calibration and the use of adaptable templating software that can accommodate various types and sizes of prostheses is essential for accurate preoperative planning (6-8).

The standard method used by templating software is the application of radio-opaque markers to calibrate the digital radiograph (9). There are concerns that positional errors of the markers could negatively affect their precision, resulting in erroneous calibration (3). Furthermore, correct positioning of external calibration markers is paramount to the reliable digital templating of component sizes (10).

The King Mark (KM) radiographic calibration method was introduced by the University of Warwick and University Hospitals Coventry and Warwickshire (UHCW) (11). It has revolutionized the reliability of radiographic magnification and consequently helped improve the accuracy of digital implant templating (12). Proposed superiority of KM calibration over traditional methods relates to the use of double calibration markers which results in improved consistency in magnification adjustment (11).

In cases of unilateral osteoarthritis (OA), preoperative templating is currently performed on either the ipsilateral pathological joint or the contralateral healthy joint, based on the preference of the surgeon. Several studies have underlined the variations that exist in patient hip morphology, that contribute to the pathogenesis of OA (13-15).

As preoperative templating takes into consideration the hip joint asymmetry, morphology and degenerative changes, logically, this could affect the accuracy when templating the diseased joint, in comparison to the healthy joint. To the best of our knowledge, no study has previously assessed whether preoperatively templating on the diseased joint influences the accuracy of implant selection in cases of unilateral OA.

The main purpose of this study was to compare preoperative templating accuracy of THA implant sizes with KM calibration using the ipsilateral diseased joint versus the contralateral healthy hip in patients with OA.

\section{METHODS}

Institutional research ethics board approval was obtained prior to commencement of this retrospective study. The study group consisted of patients undergoing primary THA for OA at our center between January 2018 and January 2020. The Corail $^{\text {TM }}$ stem (DePuy Synthes, Warsaw, IN) and Pinnacle $^{\mathrm{TM}}$ cup (DePuy Synthes, Warsaw, IN) were used in all cases. Patients who had OA on the contralateral hip, patients who underwent bilateral THA, revision surgery, cemented THA or THA after hip fracture were excluded from the study.

Data was gathered from the patients' electronic medical records. Baseline patient characteristics collected consisted of the following : gender, age, body mass index (BMI) and laterality.

Preoperatively, patients underwent anteroposterior (AP) X-rays of the pelvis with the KM calibration device. KM calibration requires the use of two markers : a radiolucent marker pad that is placed behind the pelvis as well as a marker with radio-opaque balls, which is placed in front of the pelvis (Figure 1).
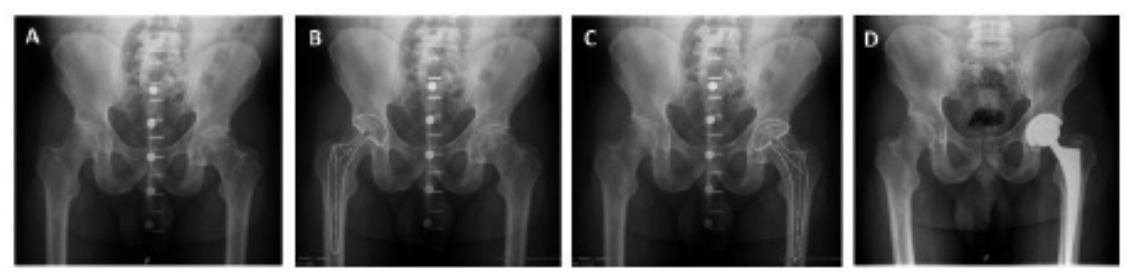

Figure 1. - From left to right: Anterior posterior X-ray of a $63 \mathrm{y} / \mathrm{o}$ male with left hip osteoarthritis with KingMark calibration device (A) who underwent a pre-operative templating on the healthy contralateral hip (B) and on the diseased operated hip (C). Post-operative X-ray us shown (D). 
The resulting X-rays were uploaded to the TraumaCad software (Voyant Health, PetachTikva, Israel) for a pre-operative digital templating. Retrospective pre-operative digital templating was performed by a single, fellowhip-trained, adult joint reconstruction surgeon (Y.W) having undergone official training by Voyant Health company instructors. Surgical reports, and actual implants sizes and characteristics were blinded from the surgeon who performed the pre-operative digital templating.

Preoperative planning was performed both on the diseased operated hip and on the contralateral healthy hip. Acetabular cup was sized so that when the template is placed with the cup at $40^{\circ} \pm 10^{\circ}$ of abduction, the medial border approximates the ilioischial line and the cup has adequate lateral bone coverage, with minimal removal of the supportive subchondral bone. Additionally, the inferior border of the cup was leveled with the inferior tear-drop line.

Stem size and neck offset were templated in order to restore both the hip center of rotation and leg length. In order to minimize any the influence of preoperative templating on final implant selection, all surgeries were performed by fellowship-trained adult joint reconstruction surgeons other than Y.W (N.S and A.G). The treating surgeons performed preoperative digital templating independently and was not made visible to the templating surgeon in this study to minimize potential bias. Final implant sizes and characteristics were collected and consisted of the following: acetabular cup size, femoral stem size and stem offset. Final implant data was kept blinded from the surgeon performing pre-operative templating for this study.

Accuracy of preoperative planning was considered adequate if a perfect match was observed or a variance of $+/-$ one size for the cup and stem, compared to the selected implant during surgery. Neck o templating was considered adequate only if that which was templated was identical to the definitive prosthesis.

Statistical analysis was performed using SPSS version 25 (IBM SPSS statistics, Chicago, IL, USA). Differences between cohorts were considered statistically significant for $\mathrm{p}<0.05$. Descriptive statistics were performed for baseline
Table I. — Patients characteristics

\begin{tabular}{|c|c|}
\hline \multicolumn{1}{|c|}{$\mathrm{n}=100$} & \\
\hline Age at surgery, years, mean (SD) & $66.57(11.45)$ \\
\hline Gender, n (\%) & $51(51.0)$ \\
\hline Male & $49(49.0)$ \\
\hline Female & $29.20(5.45)$ \\
\hline BMI, kg/m² mean (SD) & $22(22.0)$ \\
\hline BMI, n(\%) & $38(38.0)$ \\
\hline Normal weight & $40(40.0)$ \\
\hline Overweight & \\
\hline Obese & $43(43.0)$ \\
\hline Laterality, n (\%) & $57(57.0)$ \\
\hline
\end{tabular}

$\mathrm{SD}=$ standard deviation, $\mathrm{BMI}=$ Body mass index.

patient characteristics: age at surgery, gender, BMI according to WHO classification (healthy weight, overweight, obese) and surgery side. Means and standard deviations (SD) were calculated for continuous variables; frequencies and percentages were calculated for nominal variables.

Adequate planning proportions between the operated and healthy groups were compared using the McNemar test for two related groups.

\section{RESULTS}

Between January 2018 and January 2020, 540 THA were performed at our center. Of these, 100 THA used the Corail ${ }^{\mathrm{TM}}$ stem and Pinnacle ${ }^{\mathrm{TM}}$ cup and met the inclusion criteria for this study. There were 51 males and 49 females (Table I). The average age at surgery was $66.57 \pm 11.45$ years (range $32-89$ ).

The average size of the implanted acetabular cup was $52.02 \pm 3.18 \mathrm{~mm}$ (range 46-62). In the operated hip cohort, the exact acetabular cup size was predicted in $22.0 \%$ of the cases, $46.0 \%$ of the cups 
Table II. - Planning accuracy and deviation of implants

\begin{tabular}{|l|c|c|}
\hline & $\begin{array}{c}\text { Diseased hip joint } \\
\mathrm{n}(\%)\end{array}$ & $\begin{array}{c}\text { Healthy hip joint } \\
\mathrm{n}(\%)\end{array}$ \\
\hline Cup & $22(22.0)$ & $22(22.0)$ \\
\hline Exact match & $46(46.0)$ & $29(29.0)$ \\
\hline$+/-1$ size & $23(23.0)$ & $27(27.0)$ \\
\hline$+/-2$ sizes & $9(9.0)$ & $22(22.0)$ \\
\hline$>=3$ sizes & $32(32.0)$ & $32(32.0)$ \\
\hline Stem & $40(40.0)$ & $37(37.0)$ \\
\hline Exact match & $17(17.0)$ & $23(23.0)$ \\
\hline$+/-1$ size & $11(11.0)$ & $8(8.0)$ \\
\hline$+/-2$ sizes & & \\
\hline$>=3$ sizes & $73(73.0)$ & $69(69.0)$ \\
\hline Offset & & \\
\hline Exact match & & \\
\hline
\end{tabular}

were predicted within $+/$ - one size of the implanted cup and $23.0 \%$ of the cups were predicted within $+/$ - two sizes of the implanted cup. In the healthy hip cohort, the exact acetabular cup size was predicted in $22.0 \%$ of the cases, $29.0 \%$ of the cups were templated within $+/$ - one size of the cup and $27.0 \%$ of the cups were predicted within $+/-$ two sizes of the cup implanted (Table II). The overall proportion of patients who met criteria for adequate THA templating was significantly greater for those templated using the diseased hip compared to those templated using the contralateral healthy joint $(68.0 \%$ versus $51.0 \%, p<0.001)$ (Table III).

The average size of implanted femoral stems was $11.95 \pm 1.83 \mathrm{~mm}$ (range 8-16). In the diseased hip group, the exact stem size was predicted in $32.0 \%$ of the cases, $40.0 \%$ of the stems were predicted within $+/$ - one size of the implanted stem and $17.0 \%$ of the stems were predicted within +/- two sizes of the implanted stem. In the healthy hip group, the exact stem was predicted in $32.0 \%$ of the cases, $37.0 \%$ of the stems were predicted within $+/$ - one size of the implanted stem and $23.0 \%$ of the stems were predicted within $+/$ - two sizes of the implanted stem (Table II). Adequacy of preoperative templating was not found to be significantly different between both cohorts assessed, with 72.0.\% deemed acceptable in the diseased operated hip group compared to $69.0 \%$ $(\mathrm{p}=0.375)$ in the healthy hip group (Table III).

Overall, $53.0 \%$ standard offset stems $(n=51)$, $44.0 \%(n=44)$ high offset stems and 3.0\% $(n=3)$ coxa vara stems were implanted. Differences between cohorts in pre-operative templating and definitive implant offset did not reach statistical significance, with $73.0 \%$ in the operated hip group compared with $69.0 \%(p=0.289)$ in the healthy hip group (Table III).

\section{DISCUSSION}

The main finding of this study was that templating using the diseased hip is more accurate in predicting acetabular cup size compared to using the contralateral healthy hip for patients with singlesided OA undergoing THA.

The major limitation of the study was that all pre-operative templating was performed by a single surgeon which could affect the precision of preoperative planning with a repeated error. However, we believe that a single surgeon performing all templating provides internal consistency and might improve the accuracy of the results. The fact that pre-operative templating was done by a surgeon who did not perform the operation and was blinded by the implants in-situ may diminish

Table III. — Adequate planning by hip joint, entire cohort

\begin{tabular}{|l|c|c|c|}
\hline & $\begin{array}{c}\text { Adequate cup planning } \\
\mathrm{n}(\%)\end{array}$ & $\begin{array}{c}\text { Adequate stem planning } \\
\mathrm{n}(\%)\end{array}$ & $\begin{array}{c}\text { Exact offset planning } \\
\mathrm{n}(\%)\end{array}$ \\
\hline Diseased hip joint $(\mathrm{n}=100)$ & $68(68.0)$ & $72(72.0)$ & $73(73.0)$ \\
\hline Healthy hip joint $(\mathrm{n}=100)$ & $51(51.0)$ & $69(69.0)$ & $69(69.0)$ \\
\hline McNemar's test & $\mathrm{p}=0.001$ & $\mathrm{p}=0.375$ & $\mathrm{p}=0.289$ \\
\hline
\end{tabular}


observer bias. Additionally, the total number of patients participating in the study was small, nonetheless significant differences were observed.

Digital templating is an important aspect of modern THA that is routinely performed preoperatively $(6,10,16)$. Such preoperative preparation helps surgeons restore the femoral head horizontal and vertical centers of rotation, thereby minimizing limb length discrepancy (LLD) $(4,17)$.

Furthermore, templating aids in the prevention of cup malposition and helps prevent the insertion of significantly under or over-sized implants intraoperatively (18). As pre-operative planning has a major influence over the outcomes following THA, pre-operative factors that affect templating accuracy have a vital contribution to arthroplasty technique which include proper implant sizes and positioning.

The preoperative templating process is based on radiological landmarks such as the "teardrop" which is created by the superposition of the most distal part of the medial wall of the acetabulum, the tip of the anterior-posterior horn of acetabulum and the ilioischial line which represents the posterior acetabular column (19).

Such landmarks help determine proper acetabular cup size and position (18,20). Furthermore, anatomical landmarks such as the ischial tuberosities, greater and lesser trochanters are critical in the selection of neck-cut height, and in determining stem insertion depth when accounting for LLD (21).

Numerous studies have demonstrated greater templating accuracy with the aid of external markers in digital templating $(1,3,22)$. To our knowledge, no previous study has compared the accuracy of preoperative templating between the diseased and healthy hip. The aim of this study was therefore to assess whether templating on a diseased joint, compared to a healthy joint may increase the accuracy of implant selection. Evidence regarding the effect of variables such as anatomical factors, lower limb dominance, early-life hip joint dysplasia, and their effect on hip joint pathology and especially OA later in life is becoming more evident from different studies (13-15).

In their study examining measurements of femoral anteversion angle measurements using $\mathrm{CT}$, Behaeghe et al. found anatomical differences between the right and left femurs in the same patient. They concluded that patient anatomical factors should not be ignored and should be assessed preoperatively (23). Wei-Nan Zeng et al. explored anatomical differences that predispose to hip OA based on the assessment of computed tomography (CT) 3D hip reconstructions. The authors concluded that the anatomical morphology of the hip joint, such as femoral head-neck relationship, sphericity of the femoral head, acetabular coverage, and anteversion of the femoral neck and acetabulum may affect the pathogenesis of hip OA (15).

Cawley et al. described limb dominance to be an epidemiological entity and suggested the tasks of the dominant limb (e.g. propulsion) may contribute to a more rapid degeneration of the hip joint cartilage (13). A study by Philippi et al. evaluated the effect of lower limb dominance as a risk factor for femoroacetabular impingement. The authors concluded that owing to the functional asymmetry between limbs, increased pathologic forces are exerted on the dominant hip joint and proximal femoral physis (14). Findings of pelvic pronounced asymmetry around the femoral head and by extension the position and orientation of the natural center of rotation were described by Audenaert et al. The authors attributed their findings to differences in left-right dominance that may lead to inaccurate anatomical restoration of the hip in THAs (24).

The findings of these studies reflect the profound impact that anatomical variations have on hip joint morphology. These differences can lead to hip joint asymmetry and serve independently as a risk factor for the development of OA. In a patient with unilateral OA, these factors such as limb dominance or asymmetry that have been present throughout their life result in differing morphologies between the diseased joint and healthy joint.

Furthermore, acetabular degenerative changes which occurin OAsuch as osteosclerosis, osteophytes and joint space narrowing (25) are important factors contributing to the determination of cup size and positioning during surgery. Preoperative templating on the operated hip takes into consideration both the patient's basic hip joint asymmetry, degenerative changes and acetabular morphology to determine proper cup size and position (acetabular tilt and cup 
depth compared to the ilioischial line and teardrop). Taking such anatomical factors into account when templating using the diseased hip may help explain why superior templating accuracy was observed compared to using the healthy contralateral side (Table III).

Contrary to cup size, templating femoral stem and neck type in cases of end-stage OA may be more challenging. Femoral offset is known to be essential to improve function and longevity of hip arthroplasty (26). Flecher et al. found that failure to restore offset is a reason for patient dissatisfaction and significantly impairs quality of life, further emphasizing the importance of accurate preopertaive planning and intraopertaive appropriate chosen implants (17).

Numerous studies have shown that highly experienced surgeons can achieve significantly higher accuracy rates in pre-operative templating compared to less experienced individuals. Accuracy in templating precision, however, may vary according to the specific implant type (acetabulum versus femur). Holtzer et al. demonstrated that higher levels of surgeon experience lead to more precise stem size prediction in THA templating (6). Mitag et al. showed that highly experienced surgeons better predicted both final acetabular cup and stem size (27). Shichman et al. furthermore demonstrated that individual experience in preoperative digital templating plays a significant role in templating accuracy, especially in in the context of complex hip joint pathology (28).

We believe that experienced surgeons may have a better understanding of the anatomical variations associated with advanced pathologies and thus take into consideration degenerative changes derived deformities such as LLD to account for during preoperative planning $(29,30)$.

This may explain the fact that our study showed no significant difference in preoperative templating accuracy when using the diseased hip versus the healthy contralateral hip for stem size and neck offset.

\section{CONCLUSION}

Templating using the diseased hip is more accurate in predicting acetabular cup size compared to using the contralateral healthy hip for patients with single-sided OA undergoing THA.

Experienced surgeons must be aware to take into consideration hip joint asymmetry and degenerative changes seen in radiographs of OA patients during preoperative templating.

Overall, we conclude that templating on the diseased hip joint to achieve higher accuracy in predicting proper components sizes should routinely be used in patients undergoing THA for OA.

\section{REFERENCES}

1. Bourne RB, Rorabeck CH. Soft tissue balancing : the hip. J Arthroplasty. 2002 ; 17(4 Suppl 1) : 17-22.

2. Dong N, Yang C, Li SQ, Gao YH, Liu JG, Qi X. A novel digital templating methodology for arthroplasty: experience from patients with osteonecrosis of the femoral head. Hip Int. 2017 ; 27(1) : 82-6.

3. Kosashvili Y, Shasha N, Olschewski E, Safir O, White $\mathbf{L}$, Gross A, et al. Digital versus conventional templating techniques in preoperative planning for total hip arthroplasty. Can J Surg. 2009 ; 52(1) : 6-11.

4. Sariali E, Mauprivez R, Khiami F, Pascal-Mousselard H, Catonne Y. Accuracy of the preoperative planning for cementless total hip arthroplasty. A randomised comparison between three-dimensional computerised planning and conventional templating. Orthop Traumatol Surg Res. 2012 ; 98(2) : 151-8.

5. Wang Q, Xiao J, Zhu L, Zhao X, Liu Z, Wang J, et al. Acetate templating on calibrated standing digital radiograph improves accuracy of preoperative templating for total hip arthroplasty. Orthop Traumatol Surg Res. 2017 ; 103(3) : 341-7.

6. Holzer LA, Scholler G, Wagner S, Friesenbichler J, Maurer-Ertl W, Leithner A. The accuracy of digital templating in uncemented total hip arthroplasty. Arch Orthop Trauma Surg. $2019 ; 139(2)$ : 263-8.

7. Efe T, El Zayat BF, Heyse TJ, Timmesfeld N, FuchsWinkelmann S, Schmitt J. Precision of preoperative digital templating in total hip arthroplasty. Acta Orthop Belg. $2011 ; 77(5)$ : 616-21.

8. Jassim SS, Ingham C, Keeling M, Wimhurst JA. Digital templating facilitates accurate leg length correction in total hip arthroplasty. Acta Orthop Belg. 2012 ; 78(3) : 344-9.

9. Arnaout F, Dewan V, Paliobeis C. The 3-dot circle : A reliable method for safe and efficient digital templating of the acetabular component. J Orthop. $2018 ; 15(3)$ : 787-91.

10. Boese CK, Wilhelm S, Haneder S, Lechler P, Eysel P, Bredow J. Influence of calibration on digital templating of hip arthroplasty. Int Orthop. 2019 ; 43(8) : 1799-805.

11. Alnahhal A, Aslam-Pervez N, Sheikh HQ. Templating Hip Arthroplasty. Open Access Maced J Med Sci. 2019 ; 7(4) : 672-85. 
12. King RJ, Makrides P, Gill JA, Karthikeyan S, Krikler SJ, Griffin DR. A novel method of accurately calculating the radiological magnification of the hip. J Bone Joint Surg Br. 2009 ; 91(9) : 1217-22.

13. Cawley DT, Guerin SJ, Walsh J, Simpkin A, Masterson EL. The significance of hand dominance in hip osteoarthritis. Semin Arthritis Rheum. 2015 ; 44(5) : 52730.

14. Philippi MT, Kahn TL, Adeyemi TF, Maak TG, Aoki SK. Leg dominance as a risk factor for femoroacetabular impingement syndrome. J Hip Preserv Surg. 2020 ; 7(1) : 22-6.

15. Zeng WN, Wang FY, Chen C, Zhang Y, Gong XY, Zhou K, et al. Investigation of association between hip morphology and prevalence of osteoarthritis. Sci Rep. $2016 ; 6: 23477$.

16. Baxter JA, Barlow T, Karthikeyan S, Mayo DJ, King RJ. The accuracy of automatic calibration of digital pelvic radiographs using two different scale markers : a comparative study. Hip Int. 2012 ; 22(1) : 82-9.

17. Flecher X, Ollivier M, Argenson JN. Lower limb length and offset in total hip arthroplasty. Orthop Traumatol Surg Res. 2016 ; 102(1 Suppl) : S9-20.

18. Della Valle AG, Padgett DE, Salvati EA. Preoperative planning for primary total hip arthroplasty. J Am Acad Orthop Surg. 2005 ; 13(7) : 455-62.

19. O'Sullivan GS, Goodman SB, Jones HH. Computerized tomographic evaluation of acetabular anatomy. Clin Orthop Relat Res. 1992(277) : 175-81.

20. Shaarani SR, McHugh G, Collins DA. Accuracy of digital preoperative templating in 100 consecutive uncemented total hip arthroplasties : a single surgeon series. $J$ Arthroplasty. $2013 ; 28(2): 331-7$.

21. Lim SJ, Park YS. Plain Radiography of the Hip: A Review of Radiographic Techniques and Image Features. Hip Pelvis. 2015 ; 27(3) : 125-34.
22. Learmonth ID, Young C, Rorabeck C. The operation of the century: total hip replacement. Lancet. 2007 ; 370(9597) : 1508-19.

23. Behaeghe O, Van Beeck A, Dossche L, Somville J. Femoral anteversion measurement : evaluation of inter- and intraobserver reliability. Acta Orthop Belg. 2020 ; 86(1) : $17-21$.

24. Audenaert EA, Pattyn C, Steenackers G, De Roeck J, Vandermeulen D, Claes P. Statistical Shape Modeling of Skeletal Anatomy for Sex Discrimination: Their Training Size, Sexual Dimorphism, and Asymmetry. Front Bioeng Biotechnol. $2019 ; 7$ : 302 .

25. Kovalenko B, Bremjit P, Fernando N. Classifications in Brief: Tonnis Classification of Hip Osteoarthritis. Clin Orthop Relat Res. 2018 ; 476(8) : 1680-4.

26. Lecerf G, Fessy MH, Philippot R, Massin P, Giraud $\mathbf{F}$, Flecher $\mathbf{X}$, et al. Femoral offset : anatomical concept, definition, assessment, implications for preoperative templating and hip arthroplasty. Orthop Traumatol Surg Res. 2009 ; 95(3) : 210-9.

27. Mittag F, Ipach I, Schaefer R, Meisner C, Leichtle U. Predictive value of preoperative digital templating in THA depends on the surgical experience of the performing physician. Orthopedics. $2012 ; 35(2)$ : e144-7.

28. Shichman I, Factor S, Shaked O, Morgan S, Amzallag $\mathbf{N}$, Gold A, et al. Effects of surgeon experience and patient characteristics on accuracy of digital pre-operative planning in total hip arthroplasty. Int Orthop. 2020 ; 44(10) : 1951-6.

29. Carter LW, Stovall DO, Young TR. Determination of accuracy of preoperative templating of noncemented femoral prostheses. J Arthroplasty. 1995 ; 10(4) : 507-13.

30. Jung S, Neuerburg C, Kappe T, Wernerus D, Reichel H, Bieger R. [Validity of digital templating in total hip arthroplasty: impact of stem design and planner's experience]. Z Orthop Unfall. 2012 ; 150(4) : 404-8. 